\title{
Performance of international prognostic indices in plasmablastic lymphoma: a comparative evaluation
}

\author{
Nadine Hertel ${ }^{1} \cdot$ Hartmut Merz $^{2} \cdot$ Heinz-Wolfram Bernd ${ }^{2} \cdot$ Veronica Bernard $^{2} \cdot$ Axel Künstner $^{3,4} \cdot$ Hauke Busch $^{3,4}$. \\ Nikolas von Bubnoff ${ }^{1} \cdot$ Alfred C. Feller $^{2} \cdot$ Hanno M. Witte ${ }^{1,5} \cdot$ Niklas Gebauer $^{1}$
}

Received: 28 November 2020 / Accepted: 22 February 2021 / Published online: 3 March 2021

(c) The Author(s) 2021

\begin{abstract}
Purpose Plasmablastic lymphoma (PBL) is a rare and aggressive B-cell malignancy with a heterogenous clinical and prognostic spectrum, determined by multiple factors, including age, HIV- and $M Y C$-status. While there exist several validated scoring systems for diffuse large B-cell lymphoma, which incorporate basic clinical features (age, lactate dehydrogenase, sites of (extranodal) involvement, stage and performance), none of these have been systematically assessed in PBL.

Methods We determined the (age-adjusted; aa)-International Prognostic Index (IPI), revised IPI (R-IPI), and National Comprehensive Cancer Network IPI (NCCN-IPI) in a comprehensive multi-center cohort $(n=78)$ of PBL patients. Further, all indices were comparatively investigated for model quality and concordance.

Results Univariate analysis revealed significant prognostic capabilities for all indices, all of which identified a subgroup with favorable outcome. Discriminatory power between patients with less benign prognosis and especially refractory disease exhibited significant variability. Subsequently, stratified models for each risk score were compared employing corrected Akaike's information criterion (cAIC) and Harrel's concordance index (c-index). Here, the NCCN-IPI outperformed both IPI and R-IPI regarding c-index with ambiguous cAIC results, underlining its clinical utility and suggesting it for preferential use in clinical practice.

Conclusion Our current observations support the use of the IPI and its enhanced derivatives in PBL patients. There is, however, a distinct requirement for novel prognostic tools to better delineate subgroups at risk for early relapse or refractory disease as well as late relapse. A comprehensive molecular characterization of a clinically annotated cohort of PBL patients is therefore urgently warranted.
\end{abstract}

Keywords Plasmablastic lymphoma $\cdot$ IPI $\cdot$ Prognosis $\cdot$ Risk stratification

Hanno M. Witte and Niklas Gebauer shared senior authorship.

Niklas Gebauer

Niklas.Gebauer@uksh.de

1 Department of Hematology and Oncology, University Hospital of Schleswig-Holstein, Campus Lübeck, Ratzeburger Allee 160, 23538 Lübeck, Germany

2 Reference Centre for Lymph Node Pathology and Hematopathology, Hämatopathologie Lübeck, Lübeck, Germany

3 Medical Systems Biology Group, University of Lübeck, Ratzeburger Allee 160, 23538 Lübeck, Germany

4 Institute for Cardiogenetics, University of Lübeck, Ratzeburger Allee 160, 23538 Lübeck, Germany

5 Department of Hematology and Oncology, Federal Armed Forces Hospital Ulm, Oberer Eselsberg 40, 89081 Ulm, Germany

\section{Introduction}

Plasmablastic lymphoma (PBL) is a rare preterminally differentiated B-cell malignancy with features resembling both high-grade B-cell lymphoma and plasma cell malignancies (Delecluse et al. 1997; Loghavi et al. 2015; Vega et al. 2005). PBL is predominantly encountered in the context of age-related immunosenescence, secondary to iatrogenic immunosuppression or in HIV-positive patients and the clinical course is commonly aggressive with dismal overall survival (OS) (Tchernonog et al. 2017).

Patients with PBL are insufficiently represented in clinical trials, treatment approaches are heterogeneous and translational research is challenging. Nevertheless, several retrospective studies identified a number of predictors of clinical outcome including age, performance status, 
HIV- and MYC-status (Miao et al. 2020; Tchernonog et al. 2017; Witte et al. 2020).

The International Prognostic Index (IPI), the most commonly used prognostic score in aggressive B-cell lymphoma, especially in diffuse large B-cell lymphoma (DLBCL), was first developed by Shipp et al., more than 25 years ago, in the pre-rituximab era (International Non-Hodgkin's Lymphoma Prognostic Factors 1993). The analysis of more than 2000 patients with aggressive non-Hodgkin lymphoma treated with anthracycline-based regimens in the U.S., Canada and Europe led to the identification of five independent prognostic factors impacting survival: age ( $\leq 60$ vs. $>60$ years), stage (I/II vs. III/IV), number of extranodal (EN) sites ( $0-1$ vs. $\geq 2$ ), performance status (PS; $0-1$ vs. $\geq 2$ ) and serum lactate dehydrogenase (LDH; normal vs. elevated). Simultaneously, a scoring system for younger patients $<60$ years of age was developed (age-adjusted IPI). In this subgroup, ECOG (Eastern Cooperative Oncology Group) Performance-Status, stage and LDH maintained their statistically significant impact on patient outcome, thereby stratifying patients into four risk groups (score 0-3) (International Non-Hodgkin's Lymphoma Prognostic Factors 1993). Nearly fifteen years later, Sehn et al. (2007) refined the initial four risk categories (low- (score 0-1), low- intermediate- (2), high-intermediate- (3) and high- risk (4-5)) to better reflect patient outcome in the rituximab era in a more precisely defined cohort of DLBCL $(n=365)$ patients according to the WHO classification of hematopoietic and lymphoid tumors, which resulted in three distinct categories (R-IPI: 'Very good' (score 0), 'Good' (1-2) and 'Poor' (3-5)). Shortly thereafter, a pooled analysis from several prospective clinical trials, drawing data from more than 1000 patients, demonstrated the IPIs continual prognostic capabilities despite the introduction of rituximab (Ziepert et al. 2010).

More recently, in an effort to identify a subpopulation of aggressive B-cell lymphoma patients with distinctly inferior outcome (5-year OS $<50 \%$ ), despite immunochemotherapy, the IPI concept was further revised to incorporate both incremental age and LDH categories alongside the involvement of pre-defined extranodal sites (NCCN-IPI) (Zhou et al. 2014). Its proclaimed predictive superiority over the IPI/R-IPI has however been questioned, predominantly in the light of the advent of PET-based initial diagnosis and -guided therapy (El-Galaly et al. 2015). Additionally, recent reports suggest another potential refinement of the IPI through the integration of baseline beta2-microglobulin levels (GELTAMO-IPI) (Montalban et al. 2017).

However, unlike DLBCL for which there exist several validated prognostic indices, no such scoring system has been established for PBL, despite preliminary data from several retrospective investigations suggest informative applicability of the IPI in both HIV-positive and negative patients (Schommers et al. 2013; Tchernonog et al. 2017).
In the current study, we identified 78 PBL patients with available clinical data, for which in most cases (76/78) centralized hematopathological workup was available. We retrospectively evaluated clinicopathological characteristics and provide, to the best of our knowledge, the first comparison of prognostic indices ((aa)-IPI, R-IPI and NCCN-IPI) in this rare type of non-Hodgkin lymphoma.

\section{Methods}

\section{Patients and treatment}

We retrospectively reviewed our institutional hematopathological database to identify PBL patients whose biopsy specimen from initial diagnosis had been referred to the Reference center for Hematopathology University Hospital Schleswig Holstein Campus Lübeck and Hämatopathologie Lübeck for centralized histopathological panel evaluation between January 2000 and December 2018. Diagnosis was confirmed in a panel setting by three experienced hematopathologists (ACF, HM and HWB) in accordance with the current edition of the WHO classification of tumors of the hematopoietic and lymphoid tissues (Swerdlow et al. 2016). Antibodies and positivity cutoffs employed in the current study are summarized in Supplementary Table 1 and as described (Witte et al. 2020). Fluorescence in situ hybridization (FisH) for MYC was routinely performed, as described, wherever the biopsy was of sufficient size and quality (Gebauer et al. 2015).

In total, 78 consecutive patients with PBL (median age 63 years; range 26-91), were identified and assessed for clinicopathological baseline characteristics, including (age-adjusted in patients $<60$ years)-IPI, R-IPI, NCCN-IPI, ARL-IPI and GELTAMO-IPI alongside therapy as well as outcome. These characteristics of the study group are briefly summarized in Table 1, Supplementary Tables 2, 3, and 4.

This present study was approved by the ethics committee of the University of Lübeck (reference-no 18-311) and conducted in accordance with the declaration of Helsinki. Patients had given written informed consent regarding routine diagnostic and academic assessment of their biopsy specimen at the Reference center for Hematopathology and transfer of their clinical data. Most patients included in this analysis were previously investigated as part of a clinicopathological study, assessing the prognostic impact of $M Y C$ translocation status (Witte et al. 2020).

\section{Statistics}

Time to progression and overall survival (PFS, OS) were calculated from the date of initial diagnosis and censored at the date of last clinical contact. Survival (PFS and OS) 
Table 1 Clinical characteristics of the study group

\begin{tabular}{|c|c|}
\hline Characteristics & $\begin{array}{l}\text { Plasmablastic } \\
\text { lymphoma } \\
(n=78)\end{array}$ \\
\hline Age (yrs.; median + range) & $63(26-91)$ \\
\hline \multicolumn{2}{|l|}{ Sex } \\
\hline Female & $14(78 \%)$ \\
\hline Male & $64(82 \%)$ \\
\hline \multicolumn{2}{|l|}{ B-symptoms } \\
\hline Yes & $47(60 \%)$ \\
\hline No & $31(40 \%)$ \\
\hline \multicolumn{2}{|l|}{ Stage (Ann arbor) } \\
\hline $\mathrm{I} / \mathrm{II}$ & $21(27 \%)$ \\
\hline III/IV & $57(73 \%)$ \\
\hline \multicolumn{2}{|l|}{ ECOG PS } \\
\hline $0-1$ & $33(42 \%)$ \\
\hline $2-4$ & $45(58 \%)$ \\
\hline \multicolumn{2}{|l|}{ LDH } \\
\hline Normal & $17(22 \%)$ \\
\hline Elevated $\leq 3 \mathrm{x}$ & $36(46 \%)$ \\
\hline Elevated $>3 x$ & $25(32 \%)$ \\
\hline \multicolumn{2}{|l|}{ Extranodal sites } \\
\hline 0 & $12(15 \%)$ \\
\hline $1-2$ & $61(78 \%)$ \\
\hline$>2$ & $5(6 \%)$ \\
\hline Bone marrow involvement & $8(10 \%)$ \\
\hline \multicolumn{2}{|l|}{ HIV status } \\
\hline Positive & $30(39 \%)$ \\
\hline Negative & $48(62 \%)$ \\
\hline \multicolumn{2}{|c|}{ IPI (International prognostic index) } \\
\hline 0-1 (low risk) & $14(18 \%)$ \\
\hline 2 (low-intermediate risk) & $11(14 \%)$ \\
\hline 3 (high-intermediate risk) & $24(31 \%)$ \\
\hline 4-5 (high risk) & $29(37 \%)$ \\
\hline \multicolumn{2}{|c|}{ R-IPI (Revised-International prognostic index) } \\
\hline 0 (very good prognosis) & $7(9 \%)$ \\
\hline 1-2 (good prognosis) & $18(23 \%)$ \\
\hline 3-5 (poor prognosis) & $53(68 \%)$ \\
\hline \multicolumn{2}{|c|}{$\begin{array}{l}\text { NCCN-IPI (National comprehensive cancer network-International } \\
\text { prognostic index) }\end{array}$} \\
\hline $0-1$ (low risk) & $3(4 \%)$ \\
\hline 2-3 (low-intermediate risk) & $17(22 \%)$ \\
\hline 4-5 (high-intermediate risk) & $28(36 \%)$ \\
\hline 6-8 (high risk) & $30(39 \%)$ \\
\hline
\end{tabular}

ECOG PS Eastern Cooperative Oncology Group Performance Status, $L D H$ lactate dehydrogenase, yrs years

according to prognostic risk groups was initially estimated by means of the Kaplan-Meier method and univariate logrank test. Comparative analysis regarding performance of the prognostic indices was performed by employing the Harrel's concordance index (c-index) (Heller and Mo 2016) and corrected Akaike's Information Criterion (cAIC) (Akaike 1974; Sugiura 1978). While the c-index assess discriminatory power of a given statistical model (with higher values indicating superior predictive properties; $\leq 0.5$ poor model, no better than predicting an outcome than random chance; $1=$ perfect model, flawless prediction of outcome according to group allocation), the cAIC poses a means to quantify the predictive potential of statistical models upon direct comparison at low sample volumes (with lower values indicating better accuracy). A difference in cAIC values between 0 and 2 indicates the absence of significant differences in model fit while a difference between 2 and 10 suggests an increasing improvement in fit, a difference greater than 10 represents a substantial improvement in fit. All statistical investigations were conducted using GraphPad PRISM 6 and or R v4.0.2.

\section{Results}

\section{Clinical characteristics of the study group}

In total, 78 patients with PBL were retrospectively enrolled in this multi-center trial. Central histopathological review was available via the reference center for hematopathology Lübeck in 76/78 (97\%) patients. Baseline clinical characteristics are provided in Table 1. Median age of the study group was 63 years with a pronounced male predominance (64/78; 82\%). An underlying HIV infection was present in $30 / 78$ patients (39\%) and more than half of the study group presented with advanced stage disease (73\% Ann Arbor III/ IV) and reduced performance status (58\% ECOG 2-4). The vast majority of patients presented with involvement of 1-2 extranodal sites with 5/78 (6\%) patients presenting more than 2 extranodal manifestations and bone marrow infiltrates found in $8 / 78(10 \%)$ patients. An elevation in LDH levels was found in $78 \%$ of patients $(61 / 78)$ with 25 patients (32\%) showing levels $>$ three times the upper limit of norm.

While chemotherapeutic treatment approaches exhibited a substantial degree of variability, the dominant regimens of choice were $\mathrm{CHOP}$ or its derivates (55/78 patients $71 \%$ ).

\section{International prognostic indices}

Categorization according to pre-defined risk groups of all 78 patients was performed according to all three international prognostic indices. By means of the IPI $18 \%$ of patients were allocated to the low risk group, while $14 \%$ and $31 \%$ were low-intermediate or high-intermediate risk, respectively and $37 \%$ of patients were classified as high risk. In the updated R-IPI, initially proposed for aggressive B-cell lymphoma patients undergoing rituximab-containing therapy regimens, which was comparatively analyzed for internal validation and control purposes, as no improvement over the basic IPI 
was to be expected, we found $9 \%$ and $23 \%$ of patients to have a "very good" or "good" prognosis while $68 \%$ were allocated to the "poor" prognosis group.

According to the NCCN-IPI, only 3\% were low risk and $22 \%$ and $36 \%$ were low-intermediate or high-intermediate risk, respectively. A high-risk score was found in $39 \%$.

Agreement defined by weighted kappa (according to Cohen's kappa coefficient) between IPI- and NCCN-IPI was moderate (weighted $k=0.57$ ). Agreement in terms of risk category allocation was found in 54/78 (69\%) patients, while $21 / 78$ patients were grouped in adjacent risk categories. Reallocation according to the NCCN-IPI of patients initially stratified by IPI risk group is depicted in Supplementary Fig. 1. Inherent to the model design of IPI and R-IPI, due to identical individual risk factors, we observed a fixed pattern of reallocation as described (Ruppert et al. 2020; Sehn et al. 2007). Further, we calculated the AIDSrelated lymphoma (ARL)-IPI in all $\mathrm{HIV}^{+} \mathrm{PBL}$ patients for which sufficient clinical data was available (13/30) (Barta et al. 2014). Beyond the aaIPI (weighed two-fold), the ARLIPI incorporates both involvement of extranodal sites and an HIV-score comprising viral load (copies per milliliter), the number of CD4 positive T-helper cells (number per microliter) and an individual prior history of AIDS (the latter factors both weighed one-fold). Fragmentary data prevented a meaningful statistical analysis in this population. Distribution of $\mathrm{HIV}^{+} \mathrm{PBL}$ patients onto the ARL-IPI risk groups is delineated in Supplementary Table 2.

Similarly, the recent GELTAMO-IPI was only calculable in 31/78 patient due to missing baseline beta2-microglobulin levels in the majority of patients (Supplementary Table 4).

\section{Survival analysis}

At a maximum follow-up of 175 months and a median follow-up of 14 months, we observed 54 deaths and a median overall survival of 17 months. The five-year survival rate was observed to be $21.98 \%$ (95\% CI+11.06/-9.05\%). Upon
PFS analysis, we identified 58 events as well as a five-year PFS-rate of $21.14 \%$ (95\% CI + 10.376/- 8.79\%).

As depicted in Fig. 1, all three scores resulted in clinically meaningful subgroups with significant differences in OS. Estimated five-year survival according to risk groups upon employing the IPI, R-IPI and the NCCN-IPI ranged from 0 to $84.4 \%, 6.0$ to $85.7 \%$ and 0 to $100 \%$, respectively. This signifies that the widest prognostic spectrum is covered by the NCCN-IPI. Similar observations were made regarding PFS analysis. These observations are summarized in Fig. 2.

Upon comparative analysis with both IPI and R-IPI, the NCCN-IPI best identifies both a small "lowest risk" group of patients with excellent outcome as well as a "highest risk" group of patients with pronounced early therapeutic failure.

\section{Comparative analysis of model fit and concordance}

Model analysis according to both AIC as well as cAIC revealed ambiguous fit of the NCCN-IPI compared with both the R-IPI and the (aa)-IPI model. Differences were marginal, failing to reach the predefined level of significance in our minor cohort (cAIC differences <2; range: 137.8-138.6).

Analysis of concordance index, however, revealed a clinically meaningful advantage in favor of the NCCN-IPI in the discrimination of patients with poor and favorable OS (signified by the highest, yet still insufficient c-index (NCCN-IPI: 0.564; IPI: 0.523; R-IPI: 0.5). Data on model fit and concordance are summarized for OS in Table 2 and results from an univariate analysis of statistical impact of individual risk factors included in the IPI, R-IPI and the NCCN-IPI on PBL patient overall survival in addition to their individual hazard ratio are depicted in Table 3. In addition, results from both uni- and multivariate analysis of statistical impact of individual risk factors included in the IPI, R-IPI and the NCCN-IPI on PBL patient OS in the context of their respective survival model (IPI/R-IPI and NCCN-IPI) are delineated in Table 4.

In summary, our data underline the NCCN-IPI's moderate clinical utility whilst prompting its recommendation for
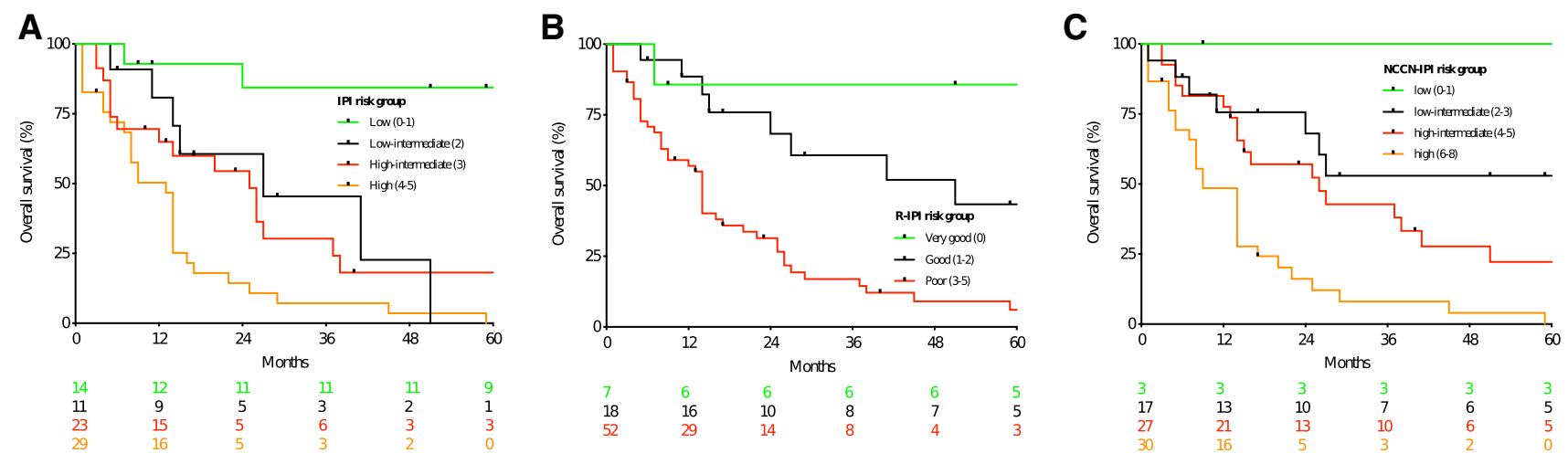

Fig. 1 Overall survival according to the IPI (a), the R-IPI (b) and the NCCN-IPI (c) 

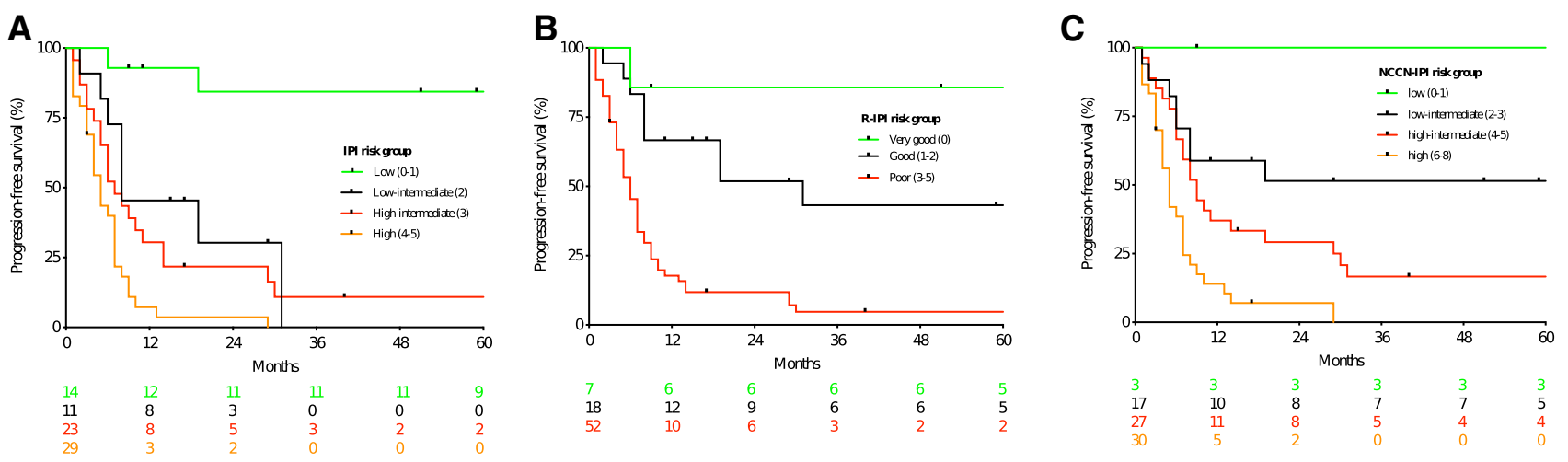

Fig. 2 Progression-free survival according to the IPI (a), the R-IPI (b) and the NCCN-IPI (c)

Table 2 Data on model fit and concordance are summarized for overall survival

\begin{tabular}{llll}
\hline Model & c-index $(95 \%$ CI) & cAIC & Hazard ratio (95\% CI) \\
\hline IPI risk group & 0.523 & 138.20 & \\
Low (0-1) & $(0.485-0.712)$ & & Reference \\
Low-intermediate (2) & & & $9.48(2.23-40.33)$ \\
High-intermediate (3) & & & $5.73(2.25-14.59)$ \\
High (4-5) & & & $7.86(3.58-17.25)$ \\
R-IPI risk group & 0.500 & 138.65 & \\
Very good (0) & $(0.409-0.640)$ & & Reference \\
Good (1-2) & & & $2.71(0.66-11.09)$ \\
Poor (3-5) & & 137.84 & $3.84(1.80-8.20)$ \\
NCCN-IPI risk group & 0.564 & & Reference \\
Low (0-1) & $(0.498-0.723)$ & & $3.33(0.45-24.40)$ \\
Low-intermediate (2-3) & & & $3.44(0.95-12.46)$ \\
High-intermediate (4-5) & & & $4.62(1.59-13.43)$ \\
High (6-8) & & & \\
\hline
\end{tabular}

preferential use in clinical practice for the time being while concomitantly characterizing the IPI as a viable option in the context of retrospective studies, facing fragmentary datasets.

\section{Discussion}

In the current study, we confirm that the original IPI clearly separates four prognostically divergent risk groups among one of the largest cohorts of PBL patients published to date regardless of HIV-status. Expectedly, no meaningful prognostic insight was gained through the application of the R-IPI (International Non-Hodgkin's Lymphoma Prognostic Factors 1993; Sehn et al. 2007). The NCCN-IPI, however, displayed superior, yet still insufficient concordance with comparable model fitting in our study group. In addition, the NCCN-IPI appears to cover the widest prognostic spectrum, allowing for a clearer distinction of patients with both most favorable and poorest OS. Similar observations were made regarding PFS analysis. Unlike recent observations in DLBCL, the NCCN-IPI reliably identified patients with both fiveyear OS- and PFS-rates below 20\% (Ruppert et al. 2020; Zhou et al. 2014). Nevertheless, both concordance and fitting measures illustrate but moderate clinical utility of all three established indices, leaving significant room for improvement.

The more detailed recognition of patient age, included in the NCCN-IPI appears to be biased, regarding the underlying HIV-status (see Table 3). Its integration into clinical prognostication models appears to be less mandatory in PBL compared with recent data taken from an integrative analysis of seven prospective trials in DLBCL (Ruppert et al. 2020). None of the established scores, however, reflect the bilateral epidemiological spectrum of PBL with elderly patients developing PBL as an indicator of senile immunosenescence 
Table 3 Univariate analysis of statistical impact of individual risk factors included in the IPI, R-IPI and the NCCN-IPI on PBL patient overall survival

\begin{tabular}{|c|c|c|}
\hline Risk factor & Hazard ratio $(95 \% \mathrm{CI})$ & $p$ value \\
\hline \multicolumn{3}{|c|}{ Age (years) (IPI/R-IPI) } \\
\hline$\leq 60$ & Reference & \multirow[t]{2}{*}{0.002} \\
\hline$>60$ & $2.356(1.353-4.105)$ & \\
\hline \multicolumn{3}{|c|}{ Age (years) (NCCN-IPI) } \\
\hline$\leq 40$ & Reference & \multirow[t]{4}{*}{0.0095} \\
\hline $41-60$ & $1.577(0.662-3.759)$ & \\
\hline $61-75$ & $2.310(1.077-4.954)$ & \\
\hline$>75$ & $1.734(0.681-4.418)$ & \\
\hline \multicolumn{3}{|l|}{ Ann arbor } \\
\hline $\mathrm{I} / \mathrm{II}$ & Reference & \multirow[t]{2}{*}{0.005} \\
\hline III/IV & $3.157(1.411-7.061)$ & \\
\hline \multicolumn{3}{|l|}{ ECOG } \\
\hline$\leq 1$ & Reference & \multirow[t]{2}{*}{$<0.0001$} \\
\hline $2-4$ & $4.603(2.477-8.554)$ & \\
\hline \multicolumn{3}{|c|}{ LDH (ULN-ratio) (IPI/R-IPI) } \\
\hline$\leq 1$ & Reference & \multirow[t]{2}{*}{$<0.0001$} \\
\hline$>1$ & $6.107(2.354-15.849)$ & \\
\hline \multicolumn{3}{|c|}{ LDH (ULN-ratio) (NCCN-IPI) } \\
\hline$\leq 1$ & Reference & \multirow[t]{3}{*}{$<0.0001$} \\
\hline $1-3$ & $3.663(1.78-7.539)$ & \\
\hline$>3$ & $5.865(2.521-13.65)$ & \\
\hline \multicolumn{3}{|c|}{ Extranodal sites } \\
\hline$\leq 1$ & Reference & \multirow[t]{2}{*}{0.035} \\
\hline$>1$ & $1.799(1.042-3.106)$ & \\
\hline \multicolumn{3}{|c|}{ Specific organ involvement* } \\
\hline No & Reference & \multirow[t]{2}{*}{0.082} \\
\hline Yes & $1.643(0.938-2.875)$ & \\
\hline
\end{tabular}

ECOG Eastern Cooperative Oncology Group Performance Status, $L D H$ lactate dehydrogenase

*Involvement of the bone marrow, central nervous system, liver or gastrointestinal system, or lung

or immunosuppressive treatment in the context of organ transplant and significantly younger PBL patients harboring an underlying acquired immune deficiency syndrome (AIDS).

Upon biphasic multivariate analysis of the individual factors of all three scoring systems (IPI and R-IPI followed by the more specific incremental variables required to calculate the NCCN-IPI) we found that the effect of stage I/ II vs. III/IV was non-independent, plausibly attributable to the inherent extranodal nature of PBL. Moreover, we found, that recognition of the specific subset of involved sites included in the NCCN-IPI was superior to the cut-off of $0-1$ vs. $>1$ extranodal sites in the (R-) IPI, whereas statistical significance and independence was lost upon incremental evaluation of LDH levels, calling into question this particular refinement of the NCCN-IPI in PBL prognostication.

In a recent meta-analysis evaluating the prognostic capabilities of IPI, R-IPI and NCCN-IPI in R-CHOP treated DLBCL patients, four out of the initial 14 trails extracted from the SEAL database had to be excluded due to missing data regarding extranodal sites of involvement (Ruppert et al. 2020). This suggests that despite all prognostic indices evaluated in this study being based on readily available clinical data, fragmentary documentation, especially in patients treated prior to the introduction or widespread implementation of the NCCN-IPI may pose an issue.

More so, established scoring systems fail to identify signs of refractory disease as well as PBL patients at risk of late relapse with sufficient statistical significance. In DLBCL, similar requirements were recently addressed successfully through the integrative analysis of clinical, molecular and cytogenetic data (Chapuy et al. 2018; Schmitz et al. 2018). Beyond recent advances regarding the prognostic role of MYC in PBL, similar data are warranted (Witte et al. 2020).

The shortcomings, inherent to such a retrospective study design, as well as the therapeutic variability, common to PBL treatment approaches, are acknowledged. This unfortunately prevented a meaningful analysis regarding the ARLIPI in the $\mathrm{HIV}^{+}$subgroup of our cohort. This model was however conceptualized in $\mathrm{CD} 20^{+}$lymphoma patients in the rituximab-era and its contributing, lymphoma-related risk factors resemble the aa-IPI which we revealed to show inferior concordance, compared to the NCCN-IPI. In prospectively assessed cohort of $\mathrm{HIV}^{+} \mathrm{PBL}$ patients it would appear preferable to investigate the addition of the ARL-IPI HIV score into the NCCN-IPI. Moreover, it would be of interest to investigate the predictive properties of the GELTAMOIPI in a cohort with sufficient information on baseline beta2-microglobulin levels (Montalban et al. 2017).

In summary, our observations support the use of the IPI and more so its enhanced derivative NCCN-IPI in PBL patients. There is, however, a distinct requirement for novel prognostic tools to better delineate subgroups at risk for early relapse or refractory disease as well as late relapse. A comprehensive molecular characterization of a clinically annotated cohort of PBL patients is therefore urgently warranted to identify additional risk factors, advance baseline prognostication and potentially treatment guidance. 
Table 4 Uni- and multivariate analysis of statistical impact of individual risk factors included in the IPI, R-IPI and the NCCN-IPI on PBL patient overall survival in the context of their respective survival model (IPI/R-IPI and NCCN-IPI)

\begin{tabular}{lccc}
\hline Risk factor & Univeriate analysis & \multicolumn{2}{l}{ Multivariate analysis } \\
& $p$ value & Hazard ratio (95\% CI) & $p$ value \\
\hline IPI/R-IPI & & & \\
Age & 0.002 & $1.820(0.994-3.334)$ & 0.052 \\
Stage I/II vs. III/IV & 0.005 & $0.753(0.276-2.054)$ & 0.579 \\
ECOG 0-1 vs. 2-4 & $<0.0001$ & $2.521(1.217-5.220)$ & $\mathbf{0 . 0 1 3}$ \\
LDH $<$ uln vs. $>$ uln & $<0.0001$ & $3.565(1.036-12.264)$ & $\mathbf{0 . 0 4 4}$ \\
Extranodal sites 0-1 vs.>1 & 0.035 & $1.621(0.910-2.888)$ & 0.101 \\
NCCN-IPI & & & \\
Age (groups 1-4) & 0.0095 & $1.260(0.905-1.754)$ & 0.171 \\
Stage I/II vs. III/IV & 0.005 & $1.035(0.390-2.745)$ & 0.944 \\
ECOG 0-1 vs. 2-4 & $<0.0001$ & $3.049(1.383-6.724)$ & $\mathbf{0 . 0 0 6}$ \\
LDH (groups 1-3) & $<0.0001$ & $1.677(0.982-2.863)$ & 0.058 \\
Specific extranodal sites* & 0.082 & $2.156(1.177-3.949)$ & $\mathbf{0 . 0 1 3}$ \\
\hline
\end{tabular}

Bold values indicate statistically significance $p \leq 0.05$

ECOG Eastern Cooperative Oncology Group Performance Status, $L D H$ lactate dehydrogenase

*Involvement of the bone marrow, central nervous system, liver or gastrointestinal system, or lung
Supplementary Information The online version contains supplementary material available at https://doi.org/10.1007/s00432-021-03580-z.

Author contributions Study concept: NG and HW; data collection: NH, $\mathrm{NG}, \mathrm{HW}, \mathrm{HM}, \mathrm{HWB}$ and VB; data analysis and creation of figures and tables: NG, HW, HB, AF, NvB and AK; initial draft of manuscript: NG; critical revision and approval of final version: all authors. All authors have read and agreed to the published version of the manuscript.

Funding Open Access funding enabled and organized by Projekt DEAL. This work was supported by grants from the Soldatentumorhilfe.

Data availability No individual participant data will be available automatically. No other documents will be available. Upon request, underlying dataset would be provided for individual participant data meta-analysis.

\section{Declarations}

Conflict of interest The authors declare no conflicts of interest.

Open Access This article is licensed under a Creative Commons Attribution 4.0 International License, which permits use, sharing, adaptation, distribution and reproduction in any medium or format, as long as you give appropriate credit to the original author(s) and the source, provide a link to the Creative Commons licence, and indicate if changes were made. The images or other third party material in this article are included in the article's Creative Commons licence, unless indicated otherwise in a credit line to the material. If material is not included in the article's Creative Commons licence and your intended use is not permitted by statutory regulation or exceeds the permitted use, you will need to obtain permission directly from the copyright holder. To view a copy of this licence, visit http://creativecommons.org/licenses/by/4.0/.

\section{References}

Akaike H (1974) A new look at the statistical model identification. IEEE Trans Automatic Control AC 19:716-723

Barta SK et al (2014) A new prognostic score for AIDS-related lymphomas in the rituximab-era. Haematologica 99:1731-1737. https ://doi.org/10.3324/haematol.2014.111112

Chapuy B et al (2018) Molecular subtypes of diffuse large B cell lymphoma are associated with distinct pathogenic mechanisms and outcomes. Nat Med 24:679-690. https://doi.org/10.1038/s4159 1-018-0016-8

Delecluse HJ et al (1997) Plasmablastic lymphomas of the oral cavity: a new entity associated with the human immunodeficiency virus infection. Blood 89:1413-1420

El-Galaly TC et al (2015) Outcome prediction by extranodal involvement, IPI, R-IPI, and NCCN-IPI in the PET/CT and rituximab era: a Danish-Canadian study of 443 patients with diffuse-large B-cell lymphoma. Am J Hematol 90:1041-1046. https://doi.org/10.1002/ ajh.24169

Gebauer N, Bernard V, Gebauer W, Thorns C, Feller AC, Merz H (2015) TP53 mutations are frequent events in double-hit B-cell lymphomas with MYC and BCL2 but not MYC and BCL6 translocations. Leuk Lymphoma 56:179-185. https://doi. org/10.3109/10428194.2014.907896

Heller G, Mo Q (2016) Estimating the concordance probability in a survival analysis with a discrete number of risk groups. Lifetime Data Anal 22:263-279. https://doi.org/10.1007/s10985-015-9330-3

International Non-Hodgkin's Lymphoma Prognostic Factors P (1993) A predictive model for aggressive non-Hodgkin's lymphoma. $\mathrm{N}$ Engl J Med 329:987-994. https://doi.org/10.1056/NEJM199309 303291402

Loghavi S et al (2015) Stage, age, and EBV status impact outcomes of plasmablastic lymphoma patients: a clinicopathologic analysis of 61 patients. J Hematol Oncol 8:65. https://doi.org/10.1186/s1304 5-015-0163-Z

Miao L, Guo N, Feng Y, Rao H, Wang F, Huang Q, Huang Y (2020) High incidence of MYC rearrangement in human immunodeficiency virus-positive plasmablastic lymphoma. Histopathology 76:201-211. https://doi.org/10.1111/his.13959 
Montalban C et al (2017) Validation of the NCCN-IPI for diffuse large B-cell lymphoma (DLBCL): the addition of beta2 -microglobulin yields a more accurate GELTAMO-IPI. Br J Haematol 176:918928. https://doi.org/10.1111/bjh.14489

Ruppert AS et al (2020) International prognostic indices in diffuse large B-cell lymphoma: a comparison of IPI R-IPI, and NCCN-IPI. Blood 135:2041-2048. https://doi.org/10.1182/blood.2019002729

Schmitz R et al (2018) Genetics and pathogenesis of diffuse large B-cell lymphoma. N Engl J Med 378:1396-1407. https://doi. org/10.1056/NEJMoa1801445

Schommers P et al (2013) Poor outcome of HIV-infected patients with plasmablastic lymphoma: results from the German AIDSrelated lymphoma cohort study. AIDS 27:842-845. https://doi. org/10.1097/QAD.0b013e32835e069d

Sehn LH et al (2007) The revised international prognostic index (R-IPI) is a better predictor of outcome than the standard IPI for patients with diffuse large B-cell lymphoma treated with R-CHOP. Blood 109:1857-1861. https://doi.org/10.1182/blood-2006-08-038257

Sugiura N (1978) Further analysts of the data by akaike' s information criterion and the finite corrections. Commun Stat Theory Methods 7:13-26. https://doi.org/10.1080/03610927808827599

Swerdlow SH et al (2016) The 2016 revision of the World Health Organization classification of lymphoid neoplasms. Blood 127:2375-2390. https://doi.org/10.1182/blood-2016-01-643569

Tchernonog E et al (2017) Clinical characteristics and prognostic factors of plasmablastic lymphoma patients: analysis of 135 patients from the LYSA group. Ann Oncol 28:843-848. https://doi. org/10.1093/annonc/mdw684

Vega F et al (2005) Plasmablastic lymphomas and plasmablastic plasma cell myelomas have nearly identical immunophenotypic profiles. Mod Pathol 18:806-815. https://doi.org/10.1038/modpa thol.3800355

Witte HM et al (2020) Clinicopathological characteristics and MYC status determine treatment outcome in plasmablastic lymphoma: a multi-center study of 76 consecutive patients. Blood Cancer J 10:63. https://doi.org/10.1038/s41408-020-0327-0

Zhou $\mathrm{Z}$ et al (2014) An enhanced International Prognostic Index (NCCN-IPI) for patients with diffuse large B-cell lymphoma treated in the rituximab era. Blood 123:837-842. https://doi. org/10.1182/blood-2013-09-524108

Ziepert M, Hasenclever D, Kuhnt E, Glass B, Schmitz N, Pfreundschuh M, Loeffler M (2010) Standard International prognostic index remains a valid predictor of outcome for patients with aggressive CD20+ B-cell lymphoma in the rituximab era. J Clin Oncol 28:2373-2380. https://doi.org/10.1200/JCO.2009.26.2493

Publisher's Note Springer Nature remains neutral with regard to jurisdictional claims in published maps and institutional affiliations. 